\title{
ÁNALISE DAS NOTIFICAÇÕES DE VIOLÊNCIA AUTOPROVOCADA POR TENTATIVA DE SUICÍDIO NO ESTADO DA PARAÍBA NO ANO DE 2014 A 2017
}

\author{
ANALYSIS OF NOTIFICATIONS OF SELF-PROMOTED VIOLENCE BY \\ ATTEMPTED SUICIDE IN THE STATE OF PARAÍBA IN THE YEAR \\ 2014 TO 2017
}

Dáfiny do Nascimento Costa ${ }^{1}$ Maria Andrelly Matos de Lima ${ }^{2}$ Lívia Mirelly Ferreira de Lima $^{3}$

RESUMO: OBJETIVO: Analisar as informações contidas nas notificações de violência segundo a lesão autoprovocada com perspectiva na tentativa de suicídio ocorrido no Estado da Paraíba no período de 2014 a 2017. MÉTODO: Trata-se de um estudo quantitativo, descritivo, observacional, cujos dados foram coletados do Departamento de Informático do Sistema Único de Saúde (DATASUS). A partir do Sistema de Informação de Agravos Notificado - SINAN e do Sistema de Informação de Mortalidade - SIM. Analisando os casos notificados comparando com os óbitos ocorridos por lesão autoprovocada. RESULTADOS: Foram registradas 1.572 vítimas de lesão autoprovocada no estado da Paraíba no período de 2014 a 2017, sendo $66 \%$ correspondendo ao sexo feminino, a faixa etária que mais cometem esse tipo de violência são os adultos jovens. Em relação aos óbitos com o Código Internacional de Doença (CID) de Lesões autoprovocadas voluntariamente, observou-se um alto número de mortalidade no sexo masculino com 78,8. O maior índice de violência autoprovocada é por envenenamento/intoxicação com $50,8 \%$ das ocorrências. CONCLUSÃO: Foi possível avaliar que ainda existem as subnotificações, através da causa morte de lesão autoprovocada voluntariamente, uma vez que as informações registradas no SINAN divergem dos dados encontrados no SIM. Todavia com a educação continuado com os profissionais que preenchem a ficha de notificação pode não só melhorar a coleta dos dados e sua digitação como também o acolhimento do paciente e de sua família.

\footnotetext{
${ }^{1}$ Acadêmica do curso de Enfermagem da Univisa, Centro Universitário da Vitória de Santo Antão. Vitória de Santo Antão, PE, Brasil.

${ }^{2}$ Acadêmica do curso de Enfermagem do CAV, Centro Acadêmico de Vitória. Vitória de Santo Antão, PE, Brasil.

${ }^{3}$ Enfermeira pela Univisa, Centro Universitário da Vitória de Santo Antão. Coordenadora de Vigilância Epidemiológica Hospitalar no município de Vitória de Santo Antão. Vitória de Santo Antão, PE, Brasil.
} 
Palavras chave: Epidemiologia. Tentativa de Suicídio e Lesão Autoprovocada. Violência.

ABSTRACT: OBJECTIVE: To analyze the information contained in the notifications of violence according to the self-inflicted injury with a perspective on the suicide attempt that occurred in the State of Paraíba from 2014 to 2017. METHOD: This is a quantitative, descriptive, observational study, whose data were collected from the Informatics Department of the Unified Health System (DATASUS). From the Notified Diseases Information System - SINAN and the Mortality Information System - SIM. Analyzing the reported cases comparing with the deaths caused by self-harm. RESULTS: 1,572 victims of self-harm were recorded in the state of Paraiba from 2014 to 2017, with 66\% corresponding to females, the age group that most commit this type of violence is young adults. In relation to deaths with the International Disease Code (ICD) of voluntarily self-inflicted injuries, there was a high number of mortality in males, with 78.8. The highest rate of self-harm is caused by poisoning / intoxication with $50.8 \%$ of the occurrences. CONCLUSION: It was possible to assess that underreporting still exists, through the cause of death from self-inflicted injury voluntarily, since the information recorded in SINAN differs from the data found in SIM. However, with continuing education with the professionals who fill out the notification form, it can not only improve data collection and typing, but also the reception of patients and their families.

Keywords: Epidemiology. Attempted Suicide and Self-Harm. Violence. 


\section{INTRODUÇÃO}

Segundo o Ministério da Saúde apud Organização Mundial de Saúde (2019), violência definida como o uso de força física ou do poder real ou em ameaça, contra si próprio, contra outra pessoa, ou contra um grupo ou uma comunidade que resulte ou tenha qualquer possibilidade de resultar em lesão, morte, dano psicológico, deficiência de desenvolvimento ou privação.

$\mathrm{Na}$ lesão autoprovocada está presente a tentativa de suicídio, suicídio, autopunição, automutilação e autoflagelação, tendo neste estudo foco na tentativa de suicídio que é um fato complexo e multidimensional, é considerado um problema de saúde pública, podendo ser ocasionado por diversos fatores como socioeconômicos, demográficos e conflitos interfamiliar. (MS, 2019; NETO, et al, 2020).

No Brasil, em 2012, foram registradas 11.821 mortes por suicídio, sendo $77,8 \%$ das ocorrências em pessoas do sexo masculino. Nas mulheres a incidência da tentativa de suicídio e maior se comparada os homens, no entanto o índice de morte por suicido é maior no sexo masculino. Um estudo realizado em 2018 apontou que o Brasil ocupava a $8^{\mathrm{a}}$ colocação mundial das mortes por suicídio. (RIBEIRO, et al, 2018).

Em 2012, nos 172 Estados membros da Organização Mundial de Saúde, foram notificadas 804.000 mortes autoinfligidas, a proporção é de 15/100.000 homens e 8,0/100.000 mulheres. Foi possível analisar também que nos países mais desenvolvidos, o suicídio é responsável por $81 \%$ dos óbitos por causas violentas entre homens e mulheres, e nos em países em desenvolvimento e subdesenvolvidos, a taxa é de $44 \%$ das mortes no sexo masculino e $70 \%$ no sexo feminino (BAHIA, et al, 2017).

A tentativa de suicídio pode ser definida como um ato interrompido antes de resultar em morte. É considerado um grave problema de saúde, pois se estima que mais de 800.000 pessoas morra de suicídio todos os anos. Os dos fatores 
desencadeantes de tentativa de suicídio nas mulheres são situações de estresse e traumáticas, com maior reflexo no período reprodutivo. (NETO, et al, 2020; SANTOS, et al, 2017).

Os problemas biológicos, ambientais, psiquiátricos, filosófico existenciais, médicos e por motivações sociais; determinantes sociais e econômicos: desemprego, baixa escolaridade, concentração de renda, exclusão social, entre outros, também são apontados como fatores de risco associados ao comportamento suicida. Dentre os fatores psiquiátricos podemos destacar depressão, esquizofrenia, humor, ansiedade, afetivo bipolar, transtornos de personalidade, alcoolismo, desesperança, solidão e comorbidades. (BAHIA, et al, 2017; MS, 2019; REISDOLFER, et al, 2015).

Um estudo citou que segundo a Organização Mundial de Saúde, a estimativa para 2020 é que haverá 1,53 milhão. O mesmo estudo analisou o custo direto e indireto no cuidado dos pacientes com tentativa de suicídio, no hospital universitário, obteve como resultado um alto custo no ambiente hospitalar, em quanto na prevenção da tentativa de suicídio há o investimento é menor. (KLEIN, et al, 2019; SGOBIN, et al, 2018).

As fichas de notificação que estão classificadas por agravos de acordo com as Doenças e Agravos de Notificação Compulsória, servem para alimentar o Sistema de Informação de Agravos de Notificação (SINAN), no caso de tentativa de suicídio a ficha notificação é a de violência interpessoal/autoprovocada, nas situações onde o meio da agressão é por envenenamento/intoxicação necessário realizar juntamente com a ficha de intoxicação exógena. A declaração de óbito alimenta o Sistema de Informações sobre Mortalidade (SIM), sendo possível a análise dos suicídios. Todas as informações são publicadas no Departamento de Informática do Sistema Único de Saúde (DATASUS) (NETO, et al, 2020).

Segundo a portaria de $\mathrm{n}^{\circ} 204$, art. $2^{\circ}$, parágrafo VI, A notificação compulsória é a comunicação obrigatória à autoridade de saúde, realizada pelos médicos, profissionais de saúde ou responsáveis pelos estabelecimentos de saúde, públicos ou privados, sobre a ocorrência de suspeita ou confirmação de doença, agravo ou evento de saúde pública, descritos no anexo, podendo ser imediata ou semanal (GM/MS, 2016). 
Se faz necessário a veracidade das informações, pois através desses sistemas é possível tomada de decisões em saúde pública. Como analise de subnotificações, e a eficácia dos planos de estratégia a saúde, relacionados as tentativas de suicídio.

\section{OBJETIVO}

Analisar as informações contidas nas notificações de violência segundo a lesão autoprovocada com perspectiva na tentativa de suicídio ocorrido no Estado da Paraíba no período de 2014 a 2017.

\section{MÉTODO}

Trata-se de um estudo quantitativo, descritivo, observacional, cujos dados foram coletados do Departamento de Informática do Sistema Único de Saúde (DATASUS). A partir do Sistema de Informação de Mortalidade - SIM e do Sistema de Informação de Agravos de Notificação (SINAN). Medindo a proporção dos casos de tentativa de suicídio e suicídio. O instrumento preenchido é a Declaração de Óbito e Ficha de Notificação de Violência, após seu preenchimento é digitado no SIM e no SINAN a Secretaria do Estado da Paraíba encaminha os dados para o Ministério da Saúde, que disponibiliza no DATASUS. Os dados foram tabulados no (Tabnet), com os seguintes indicadores no SINAN através da lesão autoprovocada: sexo, raça, escolaridade, faixa etária, tipo da violência e meio da agressão, comparando com o SIM, segundo a causa do óbito lesão autoprovocada voluntariamente: sexo e faixa etária. Foram utilizados como critérios de inclusão, todos os dados coletados, que apontaram um percentual considerável de completude, ou seja, maior que $80 \%$, para gerar uma informação fidedigna, indicador chave foi a lesão autoprovocadas, e Código Internacional de Doenças - CID10: X60- X84 Lesões autoprovocadas 
voluntariamente. Como critério de exclusão: Os indicadores que corresponderam mais de $20 \%$ de respostas ignoradas ou em branco e que estavam fora do objetivo do estudo. Os dados foram organizados em tabelas elaboradas empregando-se $o$ software Microsoft Excel 2010, produzido pela Microsoft Corporation. Sendo distribuídos em suas frequências absolutas e analisados através de percentual simples.

\section{RESULTADOS}

Tabela 1: Perfil epidemiológico das vítimas de lesão autoprovocada ocorridos no Estado da Paraíba no período de 2014 a 2017.

\begin{tabular}{lccc}
\hline \multicolumn{1}{c}{ VARÁVEIS } & CATEGORIA & $\mathbf{N}^{\mathbf{0}}$ & $\%$ \\
\hline \multirow{3}{*}{ Sexo } & Masculino & 534 & 34,0 \\
& Feminino & 1.037 & 66,0 \\
& Ignorado & 1 & 0,0 \\
\hline \multirow{4}{*}{ Faixa Etária } & $>9$ anos & 26 & 1,7 \\
& $10-14$ & 92 & 5,9 \\
& $15-19$ & 284 & 18,1 \\
& $20-29$ & 453 & 28,8 \\
& $30-39$ & 349 & 22,2 \\
& $40-49$ & 210 & 13,4 \\
& $50-59$ & 90 & 5,7 \\
Raça & 60 e mais & 65 & 4,1 \\
& Ignorada & 3 & 0,2 \\
& Amarela & 5 & 0,3 \\
& Branca & 187 & 11,9 \\
& Indigena & 4 & 0,3 \\
& Parda & 1.061 & 67,5 \\
& Preta & 34 & 2,2 \\
& Ignorado & 281 & 17,9 \\
\hline & & $\mathbf{1 . 5 7 2}$ & $\mathbf{1 0 0 , 0}$ \\
\hline
\end{tabular}

Fonte: MS/SVS/CGIAE - Sistema de Informação de Agravos de Notificação- SINAN / 2014-2017.

*Número de Casos. 
Foram registradas 1.572 vítimas de violência autoprovocada no estado da Paraíba no período de 2014 a 2017 , sendo $66 \%$ corresponde ao sexo feminino, a faixa etária mais acometida por esse tipo de violência e a dos adultos jovens, vale ressaltar que foram informadas idades menor de $>9$ anos que não correspondem a esse tipo de violência. A raça que mais se destaca com $67,5 \%$ é a parda. Não foi possível apontar a escolaridade pois $66 \%$ da informação está ignorada. (Tabela 1).

Tabela 2: Meio da agressão segundo a violência autoprovocada no Estado da Paraíba no ano de 2014 a 2017.

\begin{tabular}{|c|c|c|}
\hline Meio da agressão & $\mathbf{N}^{*}$ & $\%$ \\
\hline Força corporal/espancamento & 129 & 8,2 \\
\hline Enforcamento & 66 & 4,2 \\
\hline Envenenamento/intoxicação & 799 & 50,8 \\
\hline Objeto Contundente & 20 & 1,3 \\
\hline Substância / objeto quente & 33 & 2,1 \\
\hline Arma de fogo & 29 & 1,8 \\
\hline Ameaça & 45 & 2,9 \\
\hline Assédio sexual & 7 & 0,4 \\
\hline Estupro & 26 & 1,7 \\
\hline Outras agressões & 383 & 24,4 \\
\hline Ignorado & 35 & 2,2 \\
\hline Total & 1.572 & 100,0 \\
\hline
\end{tabular}

Fonte:/ 2014- 2017MS/SVS/CGIAE - Sistema de Informação de Agravos de Notificação- SINAN.

\section{*Número de Casos.}

É possível observar que o maior índice do meio da agressão da violência autoprovocada é por envenenamento/intoxicação com 50,8\%. (Tabela 2). Analisando o tipo de violência informado foram destacados: física, psico/moral, tortura, sexual, financeira, negligência/abandono, trabalho infantil, intervenção legal, destacando-se então a violência física com $47,8 \%$ foi e outros tipos de violências com $48,9 \%$, onde possivelmente está especificada a tentativa de suicídio. 
Tabela 3: Autor da agressão segundo a violência autoprovocada no Estado da Paraíba no ano de 2014 a 2017.

\begin{tabular}{lcc}
\hline \multicolumn{1}{r}{ Autor da agressão } & $\mathbf{N}^{*}$ & $\%$ \\
\hline Pai & 10 & 0,6 \\
Mãe & 7 & 0,4 \\
Padrasto & 3 & 0,2 \\
Cônjuge & 54 & 3,4 \\
Ex-cônjuge & 14 & 0,9 \\
Namorado & 7 & 0,4 \\
Ex-namorado & 3 & 0,2 \\
Filho & 8 & 0,5 \\
Irmão & 7 & 0,4 \\
Amigos/Conhecidos & 47 & 3,0 \\
Desconhecidos & 24 & 1,5 \\
Policial & 2 & 0,1 \\
Própria pessoa & 1.382 & 86,8 \\
Outros Vínculos & 25 & 1,6 \\
\hline Total & $\mathbf{1 . 5 9 3}$ & $\mathbf{1 0 0 , 0}$
\end{tabular}

Fonte: MS/SVS/CGIAE - Sistema de Informação de Agravos de Notificação- SINAN / 2014-2017.

\section{*Número de Casos.}

No indicador do autor da agressão, o pai, mãe, padrasto, cônjuge, ex-cônjuge, namorado, ex-namorado, filho, irmão, policial, desconhecidos, amigos/conhecidos, outros vínculos todos juntos totalizam $13,2 \%$ dos casos e tentativa provocada pela própria pessoa $86,8 \%$, o total deu maior que o número de notificações por ter sido mais de uma pessoa envolvida na agressão de alguns casos.

Segundo a evolução do caso, 90,4\% estão em branco, ocorreram 2 óbitos por violência. 
Gráfico 1: Percentual segundo ao sexo por faixa etária na causa de óbito no grande grupo CID10: X60-X84 Lesões autoprovocadas voluntariamente, ocorridos no Estado da Paraíba no período de 2014 a 2017.

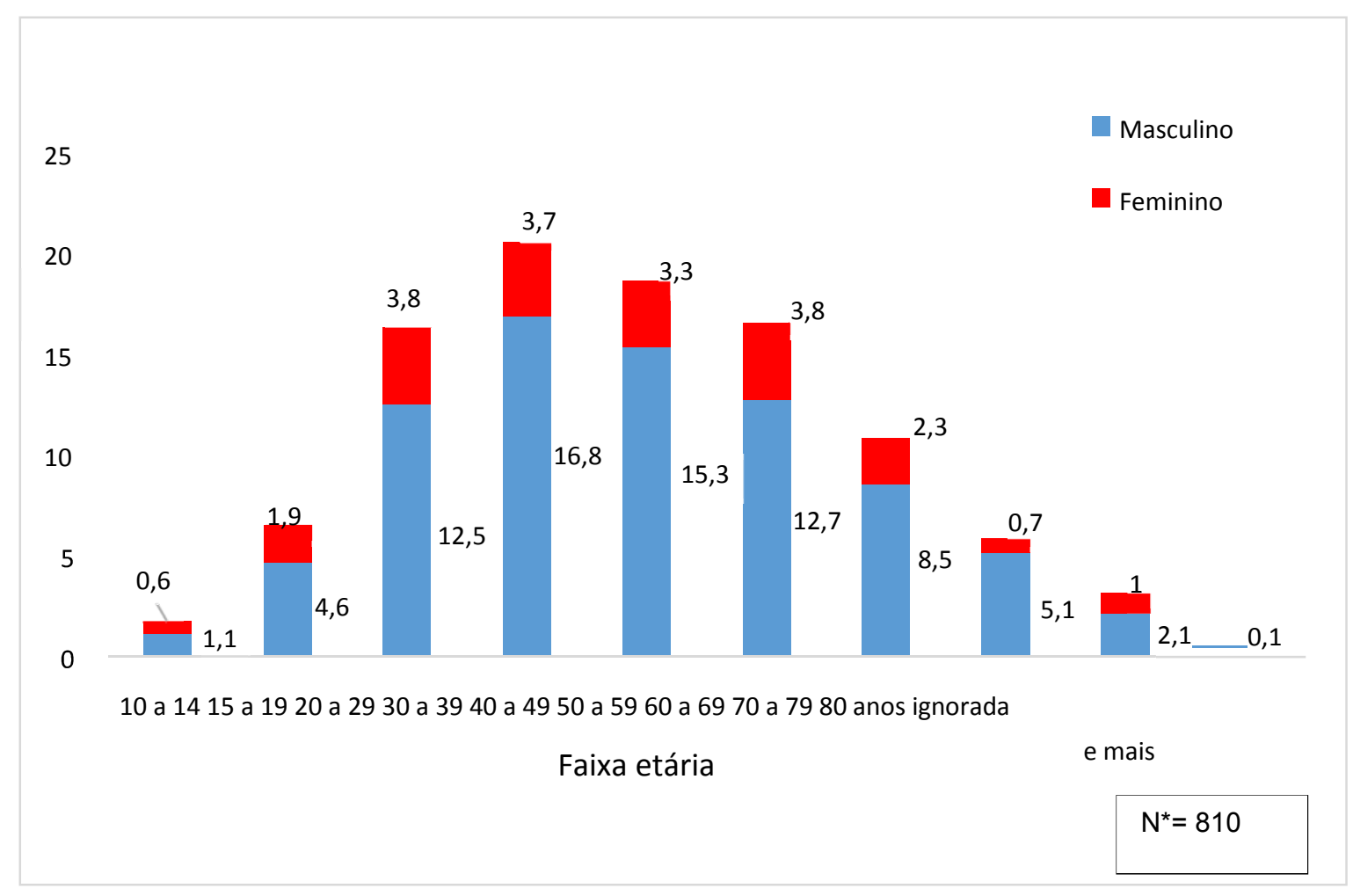

Fonte: MS/SVS/CGIAE - Sistema de Informações sobre Mortalidade - SIM / 2014 2017.

\section{*Número de Casos.}

Em relação aos óbitos com o CID de Lesões autoprovocadas voluntariamente, observou- se um alto número de mortalidade no sexo masculino com $78,8 \%$, comparado aos casos notificados do sexo feminino na tentativa de suicídio, vale ressaltar que essas causas de lesões autoprovocadas voluntariamente estão relacionadas com tentativa de suicídio, suicídio, autopunição, automutilação e autoflagelação, segundo a definição do guia de vigilância em saúde, 2019. 


\section{DISCUSSÕES}

Observou-se na analise de 1.572 casos de violência autoprovocada, que a maior porcentagem ocorre no sexo feminino tendo prevalência na faixa etária de adulto jovem, sendo envenenamento/intoxicação o meio da agressão com maior frequência. Ao analisar as informações foram observadas possíveis falhas no preenchimento da ficha de notificação ou da digitação da mesma.

Uma pesquisa realizada no Distrito Federal apontou que não foi possível analisar a ficha de notificação de Violência Interpessoal e Autoprovocada, pelo não preenchimento do documento, como o não fornecimento de dados essenciais, esse resultado vai de encontro com a análise desse estudo pelo fato de ter muitas informações que contrapõem para casos de lesões autoprovocada, de acordo com sua definição no guia de vigilância em saúde, que orienta sobre o preenchimento da ficha de notificação (BAÉRE, ZANELLO, 2018; MS, 2019).

No tipo de violência identificamos $50,8 \%$ dos casos por envenenamento/intoxicação, enforcamento $4,2 \%$, arma de fogo $1,8 \%$ objeto contundente $1,2 \%$, o somatório desses valores deveria estar em conformidade com os achados do percentual do item outros uma vez que ele é utilizado para sinalizar a tentativa de suicídio, sendo esses dados incompatíveis ao cruzar os indicadores. Encontramos divergências em relação à violência sexual que foi descrita como uma lesão autoprovocada.

Ao analisar os dados de mortalidade pelo Sistema de Informação sobre Mortalidade obtivemos como resultado 810 óbitos por lesão autoprovocada voluntariamente, desses $78,8 \%$ ocorreram com pessoas do sexo masculino com faixa etária adulto jovem, podendo ser observado que esses casos não foram compulsoriamente notificados na ficha de violência por suicídio. Estudos realizados sobre essa temática também aponta que a incidência dos óbitos por suicídio é mais elevada no sexo homens. (KLEIN, et al, 2020; MINAYO, et al, 2017; SANTOS, et al, 2018; FILHO, et al, 2019). 
Os dados encontrados em relação às lesões autoprovocadas aponta que as mulheres são mais notificadas com $66 \%$, prevalência esta também pontuada em um estudo realizado nas capitais do Brasil, onde o resultado das lesões autoprovocadas é superior a 39,8\% nas mulheres (BAHIA, et al, 2017; RIBEIRO, et al, 2018; FELIX, et al, 2017).

O meio da agressão mais usual é por envenenamento/intoxicação, vários estudos trazem como resultados de tentativa de suicídio, o maior número é entre mulheres e por meio do uso de medicamentos e/ou substancias que resultam na intoxicação exógena, sendo justificada por alguns estudos a facilidade no acesso de medicamentos, assim fazem o uso abusivo sem planejar o suicídio, ao contrário dos homens, os mesmos cometem o próprio suicídio de maneira mais letal, como no caso do enforcamento (BAHIA, et al, 2017; RIBEIRO, et al, 2018; FELIX, et al, 2017).

Nesse estudo não foi possível avaliar a renda das pessoas, como também não foi possível identificar o grau de escolaridade, que se faz necessário analisar o grau de instrução, percebendo assim a busca pelo auxílio de um profissional qualificado nessa área de saúde mental. Todavia a Organização Mundial de Saúde, afirma que $78 \%$ dos casos de suicídio ocorrem na população que tem uma renda baixa (RIBEIRO et al. apud OMS, 2020).

Se faz necessário uma identificação dos riscos de eventos suicidas, para realizar a promoção e educação da saúde mental, com a finalidade de reduzir as taxas de incidência de eventos de suicídio. O desenvolvimento dessas atividades é de competência multiprofissional (enfermeiro, psicólogo, psiquiatra), que envolve setores como gestão, Vigilância Epidemiológica do Município, Estratégia de Saúde da Família, Núcleo de Assistência a Saúde da Família e o Centro de Atenção Psicossocial (NETO, et al, 2020).

É fundamental uma capacitação desses profissionais que compõe essas equipes, que lhe dá diretamente com a vítima, como também dos profissionais que atuam nos hospitais e nas unidades de pronto atendimento, para a realização da notificação compulsória de violência autoprovocada, que é de caráter imediata. Através da notificação o profissional da Estratégia de Saúde da Família, resgata as informações essenciais para analisar a situação de sua área, e os riscos em que as 
vítimas provocam o suicídio, dessa forma realizando uma promoção à saúde mental de forma eficaz (KLEIN, et al, 2020).

\section{CONCLUSÃO}

Após traçar o perfil da violência por tentativa de suicídio e por suicídio, segundo a lesão autoprovocada, observou-se a prevalência das mulheres com o maior número de tentativas de suicídio por abuso de medicações, já em relação ao suicídio as o maior percentual de vítimas são os homens, que correspondem aproximadamente $79 \%$ dos óbitos por sempre buscarem um meio letal para que não haja falha no seu plano de morte.

Vale ressaltar que é possível prevenir esses óbitos por violência autoprovocada, por meio das politicas publicas e redes de apoio, todavia é importante avaliar a resolutividade dos serviços de Centro de Atenção Psicossocial, além de fornecer estimativas que auxiliam na tomada de decisões e no planejamento de políticas públicas.

Diante da análise dos dados viu-se a necessidade de treinamento com os profissionais de saúde, tanto no preenchimento ficha, quanto em quais situações realizar a notificação, e ressaltar a importância da digitação das fichas no sistema de forma correta, pois as inconsistências dos dados cruzados dificultou a análise dos mesmos, não podendo fechar com uma informação fidedigna dos indicadores. Foi possível avaliar que ainda existem as subnotificações, através da causa morte de lesão autoprovocada voluntariamente, uma vez que as informações registradas no SINAN divergem dos dados encontrados no SIM.

Acredita-se que para melhor redução dos casos de violência autoprovocada, é necessário uma ampliação na especialização dos profissionais que atuam diariamente na Rede de Atenção à Saúde. Visto que tendo um acolhimento diferenciado a essa população com tendência a tentar contra sua própria vida, é capaz de mudar toda essa trajetória, encaminhando para centro de especialidade que cuidem desses usuários. 
O enfermeiro possui um papel muito importante na intervenção dessa temática, visto que o profissional está em toda porta de entrada onde o paciente suicida pode comparecer, e ser acolhido pelo enfermeiro devidamente capacitado que irá oferecer uma escuta terapêutica, avaliando as angustias e necessidades dos usuários dos serviços de saúde, para serem encaminhados às unidades especializadas pra receberem esses casos. Desta forma se faz necessário que o profissional receba uma educação continuada frente à problemática, para melhor acolher não só o paciente como também a família.

\section{REFERÊNCIAS BIBLIOGRÁFICAS}

BAÉRE, F. ZANELLO V. O gênero no comportamento suicida: Uma leitura epidemiológica dos dados do Distrito Federal. Estudos de psicologia, 23(2), 2018.

BAHIA, C. et al. Lesão autoprovocada em todos os ciclos da vida: perfil das vitimas em serviços de urgência e emergência de capitais do Brasil. Ciência e Saúde Coletiva, RJ. 9(22), 2017.

Departamento de Informático do Sistema Único de Saúde (DATASUS).

FELIX, T. et al. Risco para violência autoprovocada: prenuncio de tragédia, oportunidade de prevenção. Ver electrónica trimestral de enfermería. (53), 2017.

FILHO, E. et al. O suicídio no estado de Tocantins. Ver Eletrônica Acervo Saúde. 11(12), 2019.

KLEIN, V. SILVA, W. OLIVEIRA, J. Epidemiologia do suicídio no Brasil entre os anos de 2011 e 2016. Revista de Patologia do Tocantins. 4(6), 2020.

MINAYO, M. FIGUEIREDO, A. MANGAS, R. O comportamento suicida de idosos. Physis ver de saúde coletiva, RJ. 27(4), 2017.

Ministério da Saúde. Guia de vigilância em Saúde. Brasília-DF. 2019.

NETO, P. et al. Tentativa de suicídio, transtorno de estresse pós-traumático e fatores associados em mulheres do Recife. Ver Bras Epidemiol, (23), 2020.

RIBEIRO, N. et al. Análise da tendência temporal do suicídio e de sistemas de informações em saúde em relação às tentativas de suicídio. Texto Contexto Enferm. 27(2), 2018.

SANTOS, E. BARBOSA, I. SEVERO, A. Análise espaço-temporal da mortalidade por suicídio no Rio Grande do norte, Brasil, no período de 200 a 2015. Ciência e Saúde Coletiva. 25(2), 2020.

SGOBIN, S. et al. Custo direto e indireto de tentativas de suicídio em um hospital geral: estudo de custo de doença. São Paulo Med J. 133(3), 2015. 